\title{
O Consentimento e a Recusa Esclarecidos na Cirurgia Endonasal Avançada: O Dilema Ético do Sacrifício do Olfacto na Cirurgia da Rino- Sinusite Crónica com Pólipos
}

\author{
Informed Treatment Consent and Refusal in Advanced Endonasal Surgery: The \\ Ethical Dilemma of Olfaction Sacrifice in Surgery for Chronic Rhinosinusitis \\ with Polyposis
}

\author{
João SUBTIL ${ }^{1}$, João Pedro ARAÚJO¹, José SARAIVA , Alberto SANTOS ${ }^{1}$, Paulo VERA-CRUZ², João PAÇO², Diogo \\ PAIS $^{3}$ \\ Acta Med Port 2015 Jul-Aug;28(4):513-516
}

\section{RESUMO}

Introdução: Na rino-sinusite crónica com pólipos, ou polipose naso-sinusal, o olfacto está frequentemente afectado de forma significativa, e é reconhecido o impacto importante deste sintoma na qualidade vida. A resolução por cirurgia endonasal nos casos de resistência à terapêutica médica máxima é uma opção para minorar os sintomas, mas a sua extensão é alvo de controvérsia, sendo que uma cirurgia mais radical, como a nasalização, controla melhor a doença com menos recidivas, mas pode comprometer mais o olfacto. A decisão da extensão da cirurgia deve ser partilhada com o doente, devendo o esclarecimento informado ser tão completo quanto possível quanto às expectativas de controlo da doença como à resolução da hipósmia.

Material e Métodos: Revisão bibliográfica e discussão por quadro de peritos.

Resultados e Discussão: Baseando-nos em revisão de literatura, apresentamos uma proposta de elementos a incluir no esclarecimento cirúrgico, de modo a contemplar de forma abrangente as limitações da cirurgia no que diz respeito ao sintoma anósmia, bem como das diferentes opções e suas consequências quanto à sua radicalidade, focando a atenção no ponto de vista da ética médica. Conclusão: A decisão da extensão da cirurgia deve ser partilhada com o doente, devendo o esclarecimento informado ser tão completo quanto possível quanto às expectativas de controlo da doença como à resolução da hipósmia.

Palavras-chave: Alterações do Olfacto; Consentimento Informado; Ética Médica; Pólipos/cirurgia; Procedimentos Cirúrgicos Otorrinolaringológicos; Recusa ao Tratamento.

\section{ABSTRACT}

Introduction: Olfaction is frequently affected in chronic rhino-sinusitis with polyposis and has been recognised to have important impact on quality of life. Surgical resolution on cases of maximal medical therapy failure is an option to relieve symptoms, with debates as to how extensive surgery should be. A more radical approach will achieve better disease control with less relapse, but can also compromise olfaction. This decision about a more radical surgical approach should be shared with the patient. Thorough informed consent regarding disease control and hyposmia should be taken.

Material and Methods: Literature review and consultation with a board of experts.

Results and Discussion: We propose some elements to be included in the informed consent discussion, in order to broadly address the surgical limitations regarding anosmia as a frequent complaint, as well as the different options and their associated consequences. Conclusion: Radical surgery decision making should be shared with the patient and the informed consent should be as thorough as possible regarding disease control and hyposmia resolution.

Keywords: Ethics, Medical; Informed Consent; Nasal Polyps/surgery; Olfaction Disorders; Otorhinolaryngologic Surgical Procedures; Treatment Refusal.

\section{INTRODUÇÃO}

A cirurgia endonasal tem sido alvo de grandes desenvolvimentos nos últimos anos. Depois de Messerklinger nos anos setenta do século passado ter introduzido o uso de endoscópios e do conceito de cirurgia funcional endonasal, ${ }^{1}$ o tratamento cirúrgico da doença nasal tem sido progressivamente encarado de forma mais conservadora. Entende-se desde então que as estruturas etmoidais em torno do meato médio formam um complexo funcional integrado, designado vulgarmente como complexo osteo-meatal, para onde drenam os seios maxilar e frontal e grande parte das células etmoidais. Por ser assim um confluente funcional, qualquer patologia anatómica ou inflamatória com tradução em compromisso desta área vai ter consequências a montante nas referidas cavidades sinusais, levando assim a compromisso da sua drenagem e arejamento, o que em última análise pode levar à inflamação destas cavidades, ou seja a sinusite.

Este conceito levou por sua vez à inovação no tratamento cirúrgico, que se tem afastado nestes anos da mera ablação de tecido inflamatório, para procurar antes a repermeabilização do meato médio e da drenagem sinusal, tendo-se estendido a toda a patologia inflamatória

\footnotetext{
1. Serviço de Otorrinolaringologia. Hospital Cuf Descobertas. Lisboa. Portugal.

2. Serviço de Otorrinolaringologia. Hospital Cuf Infante Santo. Lisboa. Portugal.

3. Faculdade de Ciências Médicas. Universidade Nova de Lisboa. Lisboa. Portugal.

$\bowtie$ Autor correspondente: João Subtil. joaosubtil@gmail.com

Recebido: 23 de Setembro de 2014 - Aceite: 16 de Fevereiro de 2015| Copyright $\odot$ Ordem dos Médicos 2015
} 
exceptuando a rino-sinusite crónica com pólipos.

\section{MATERIAL E MÉTODOS}

Recorremos a revisão bibliográfica de fontes da Especialidade de Otorrinolaringologia e de Ética Médica para sustentar a discussão de aspectos controversos no tratamento da polipose naso-sinusal, designadamente quanto à intenção curativa da intervenção cirúrgica, à importância da anósmia/hipósmia antes e depois da cirurgia, e finalmente, quanto à inclusão do sintoma "olfacto" na discussão do consentimento informado.

\section{RESULTADOS E DISCUSSÃO}

\section{Intenção da cirurgia na polipose nasal - curativa ou pa-} liativa?

A rino-sinusite crónica com pólipos corresponde a cerca de $20 \%$ do total de casos de rino-sinusite crónica ${ }^{2}$ e tende a ter um prognóstico mais reservado do que as restantes formas de rino-sinusite. Aqui a inflamação é mais exuberante, com uma resposta hipertrófica, e as recidivas após a cirurgia muito mais frequentes, ${ }^{2}$ o que leva a que não haja ainda consenso sobre o seu tratamento cirúrgico, ${ }^{3}$ sobretudo no que toca à sua extensão. Estes doentes são os que maior melhoria de qualidade de vida reportam, independentemente do tipo de cirurgia, ${ }^{2}$ quando comparados com casos de rino-sinusite sem pólipos, o que olhando de outro ponto de vista, significa que a doença é um fardo tão importante na qualidade de vida que qualquer intervenção traz uma melhoria muito importante.

O que é controverso entre os diversos autores é a indicação para remover as zonas onde se formam mais pólipos, que são o corneto médio e o tecto etmoidal, o que leva a um compromisso iatrogénico do olfacto. Dito de outra forma, se a intenção for curativa, alguns autores argumentam que deve ser removido o corneto médio com possível sacrifício do olfacto, procedendo então a uma etmoidectomia radical, por oposição à etmoidectomia funcional em que se poupam estas estruturas. ${ }^{4,5}$

Efectivamente, tem sido demonstrada a eficácia da etmoidectomia radical no controlo da recorrência das queixas, na melhoria funcional e na diminuição da necessidade de cirurgia de revisão. ${ }^{4,5}$ No entanto, para muitos autores a opção radical significa igualmente a amputação de pelo menos uma parte importante do aparelho olfactivo. ${ }^{4,5}$

\section{Importância da anósmia na polipose nasal - antes e de- pois da cirurgia:}

Globalmente, o impacto da rino-sinusite crónica na qualidade de vida é grande, sendo conhecido que é mesmo superior ao da insuficiência cardíaca crónica. ${ }^{6} \mathrm{E}$ é igualmente conhecido que este impacto melhora com a cirurgia, mesmo quando é analisado cada sintoma separadamente. ${ }^{7}$ No entanto a hipósmia é um sintoma que obtém melhorias modestas com a cirurgia, ${ }^{7}$ sendo simultaneamente muito comum, manifestando-se em cerca de $65 \%$ a $80 \%$ das rino-sinusites crónicas. ${ }^{8}$

A hipósmia é uma limitação sensorial frequentemente desprezada por médicos e pela sociedade em geral, mas que implica um impacto severo na qualidade de vida. ${ }^{9}$ É um sentido que é importante para avaliar a qualidade de alimentos, para alertar para fumos tóxicos, gás ou fogo, bem como, e não menos importante, para funções hedonísticas, ligadas ao paladar de alimentos ou bebidas e ao reconhecimentos de perfumes, fragrâncias e cheiros. Sem olfacto é difícil completar aceitavelmente tarefas diárias como cozinhar, cuidar da higiene pessoal ou cuidar de crianças ou de familiares dependentes, ou como degustar uma refeição.

O impacto na qualidade de vida traduz-se indirectamente em depressão mais severa, perda de peso, anorexia, e compromisso do bem-estar psicológico. ${ }^{10}$ Estudando directamente questionários de avaliação de qualidade de vida obtemos o mesmo resultado, ${ }^{9,11}$ revelando que pelo menos $33 \%$ dos doentes com hipósmia apresentam 'problemas severos' e redução importante da qualidade de vida.

Em regra, a rino-sinusite com polipose tem indicação cirúrgica quando a terapia médica máxima falhou, mantendo-se um impacto importante na qualidade de vida. ${ }^{3} A$ hipósmia é uma das queixas mais referidas, como vimos acima, e, naturalmente, o doente quer ver esse sintoma resolvido a par dos restantes. Como a cirurgia pode afectar o olfacto, ${ }^{4}$ este assunto deve ser um tema a abordar na discussão do consentimento esclarecido.

\section{Inclusão do compromisso do olfacto no consentimento esclarecido - considerações éticas:}

O processo de colheita do consentimento esclarecido é relativamente recente na História da Medicina, ${ }^{12}$ tendo sido desenvolvido nos últimos 50 anos, e reflecte o reconhecimento do princípio da autonomia ou da liberdade de escolha da pessoa no que ao tratamento diz respeito. Esta escolha só é livre e autónoma se o próprio compreender as razões e as consequências de ambos a doença e o tratamento, e cabe ao médico esclarecer o seu interlocutor de forma adequada e adaptada.

Segundo Antunes, são necessários três elementos para que o consentimento seja considerado livre e esclarecido: competência na esfera da decisão; informação apropriada; e inexistência de coerção. "Competência para decidir implica que o paciente seja capaz de compreender a informação, de decidir relativamente às escolhas possíveis e de comunicar a sua decisão". ${ }^{13}$

A opção entre diversas formas de tratamento propostas, ou mesmo a opção por não ser tratado (a recusa esclarecida) é tomada pelo próprio combinando o que entende como ganho e perda em cada destas opções e ponderando a sua importância. O peso desta importância é sempre pessoal, e radica em bases individuais ou contextuais, cognitivas ou culturais, e muitas vezes escapam à compreensão do médico, que no entanto as aceita com a naturalidade da sua obrigação profissional. Por esta razão, essa escolha deve/ tem que ser do doente.

Mais do que incluir o doente na decisão, este processo promove a adesão do doente a toda a extensão da responsabilidade da escolha, incluindo a compreensão de 
possíveis resultados negativos ou complicações. ${ }^{14}$ Facilita assim a aceitação de um resultado insuficiente, no caso de se optar por uma forma de resolução cirúrgica menos radical com intenção de evitar possíveis complicações.

\section{Proposta de esclarecimento sobre o olfacto no pro- cesso de recolha de consentimento esclarecido para cirurgia}

$\mathrm{Na}$ rino-sinusite crónica com pólipos, o esclarecimento das opções cirúrgicas deve assim ser abrangente. Quanto à patologia e causa da hipósmia, deve-se dar a informação de que este sintoma tem duas causas - a obliteração mecânica do epitélio olfactivo pelos pólipos, que impede o trânsito do ar pelos receptores olfactivos; e a alteração da mucosa por inflamação crónica que diminui a sua função sensorial. ${ }^{15}$ Depois da falência do tratamento médico máximo, deve propor-se a opção cirúrgica, e devemos sempre mencionar que este sintoma pode não melhorar de forma significativa. ${ }^{7}$ Ou seja, se a única preocupação do doente for a restauração do olfacto, este pode compreensivelmente optar por recusar o tratamento.

Quanto à extensão da cirurgia, devemos igualmente esclarecer que uma cirurgia menos radical pode ser menos eficaz e ter mais recidivas com formação de pólipos e maior probabilidade de ser necessária reintervenção, ${ }^{3}$ mas uma intervenção mais radical implica um maior risco de persistência da hipósmia, ou mesmo anósmia após a cirurgia, ainda que um estudo refira que os resultados podem ser sobreponíveis. ${ }^{15}$

Deve ainda ser informado que o melhor resultado depende da manutenção da terapêutica corticóide tópica nasal crónica, ${ }^{3}$ o que significa que a cirurgia não pode ser considerada como curativa.

Entendemos que o consentimento deve ser oral por considerarmos que o risco neste tipo de cirurgia não é 'superior ao risco mínimo de uma intervenção de rotina' deste tipo, como exposto já, e não se encontra descrito em nenhuma das circunstâncias em que legalmente é especificamente imposto o consentimento escrito. ${ }^{16} \mathrm{Em}$ Portugal vigora o princípio da 'liberdade declarativa', ${ }^{16}$ sendo utilizada a forma de um termo de responsabilidade assinado pelo doente após esclarecimento oral. A extensão deste esclarecimento e seu conteúdo são muito variáveis entre instituições, embora inclua frequentemente esclarecimento oral do procedimento, sua indicação, evolução previsível no período pós-operatório e complicações possíveis. Parece-nos importante, como exposto, que nesta fase do esclarecimento seja incluído o possível compromisso do olfacto.

Apenas assim, desta forma completa, no que toca ao sintoma hipósmia, se pode considerar o processo de decisão verdadeiramente esclarecido.

\section{CONCLUSÃO}

$\mathrm{Na}$ rino-sinusite crónica com pólipos o olfacto está frequentemente afectado de forma significativa, e é reconhecido o impacto importante deste sintoma na qualidade vida. A resolução cirúrgica nos casos de resistência à terapêutica médica máxima é uma opção para minorar os sintomas, mas a sua extensão é alvo de controvérsia, sendo que uma cirurgia mais radical controla melhor a doença com menos recidivas, mas pode comprometer mais o olfacto. A decisão da extensão da cirurgia deve ser partilhada com o doente, devendo o esclarecimento informado ser tão completo quanto possível quanto às expectativas de controlo da doença como à resolução da hipósmia.

\section{CONFIDENCIALIDADE DOS DADOS}

Os autores declaram ter seguido os protocolos do seu centro de trabalho acerca da publicação dos dados de doentes.

\section{CONFLITOS DE INTERESSE}

Os autores declaram que não houve conflitos de interesse na realização deste trabalho.

\section{FONTES DE FINANCIAMENTO}

Este trabalho não recebeu qualquer contribuição, subsídio ou bolsa.

\section{REFERÊNCIAS}

1. Husain Q, Patel SK, Soni RS, Patel AA, Liu JK, Eloy JA. Celebrating the golden anniversary of anterior skull base surgery: reflections on the past 50 years and its historical evolution. Laryngoscope. 2013;123:64-72.

2. Poetker DM, Mendolia-Loffredo S, Smith TL. Outcomes of endoscopic sinus surgery for chronic rhinosinusitis associated with sinonasal polyposis. Am J Rhinol. 2007;21:84-8.

3. Fokkens WJ, Lund VJ, Mullol J, Bachert C, Alobid I, Baroody F. European Position Paper on Rhinosinusitis and Nasal Polyps 2012. Rhinol Suppl. 2012;1-298.

4. Jankowski R, Pigret D, Decroocq F, Blum A, Gillet P. Comparison of radical (nasalisation) and functional ethmoidectomy in patients with severe sinonasal polyposis. A retrospective study. Rev Laryngol Otol Rhinol. 2006;127:131-40.

5. Masterson L, Tanweer F, Bueser T, Leong P. Extensive endoscopic sinus surgery: does this reduce the revision rate for nasal polyposis? European archives of oto-rhino-laryngology. 2010;267:1557-61.

6. Gliklich RE, Metson R. The health impact of chronic sinusitis in patients seeking otolaryngologic care. Otolaryngol Head Neck Surg. 1995;113:104-9.

7. Chester AC. Symptom outcomes following endoscopic sinus surgery.

Curr Opini Otolaryngol Head Neck Surg. 2009;17:50-8.

8. Rudmik L, Smith TL. Olfactory improvement after endoscopic sinus surgery. Curr Opin Otolaryngol Head Neck Surg. 2012;20: 29-32.

9. Miwa T, Furukawa M, Tsukatani T, Costanzo RM, DiNardo LJ, Reiter ER. Impact of olfactory impairment on quality of life and disability. Arch Otolaryngol Head Neck Surg. 2001;127:497-503.

10. Deems DA, Doty RL, Settle RG, Moore-Gillon V, Shaman P, Mester AF, et al. Smell and taste disorders, a study of 750 patients from the University of Pennsylvania Smell and Taste Center. Arch Otolaryngol Head Neck Surg. 1991;117:519-28.

11. Croy I, Nordin S, Hummel T. Olfactory disorders and quality of life -an updated review. Chem Senses. 2014;39:185-94.

12. Esperança Pina JA. Relação Médico-Doente. In: Ética, Deontologia e Direito Médico. Lisboa: Lidel Edições Técnicas; 2013.

13. Antunes A, Nunes R. Consentimento Informado na prática clínica. Arq Med. 1999;2:121-7.

14. Kern EB. The preoperative discussion as a prelude to managing a complication. Arch Otolaryngol Head Neck Surg. 2003;129:1163-5.

15. Jankowski R, Bodino C. Olfaction in patients with nasal polyposis: effects of systemic steroids and radical ethmoidectomy with middle 
turbinate resection (nasalization). Rhinology. 2003;41:220-30.

16. Entidade reguladora da saúde [página de internet]. Consentimento informado - Relatório final Maio de 2009 [consultado 2014 Nov 08]
Disponível em https://www.ers.pt/uploads/writer file/document/73/ Estudo-Cl.pdf. 


\section{O Consentimento e a Recusa Esclarecidos na Cirurgia Endonasal Avançada: O Dilema Ético do Sacrifício do Olfacto na Cirurgia da Rino-Sinusite Crónica com Pólipos}

Acta Med Port 2015:28:513-516

Publicado pela Acta Médica Portuguesa, a Revista Científica da Ordem dos Médicos

Av. Almirante Gago Coutinho, 151

1749-084 Lisboa, Portugal.

Tel: +351218428215

E-mail: submissao@actamedicaportuguesa.com

www.actamedicaportuguesa.com

ISSN:0870-399X | e-ISSN: 1646-0758

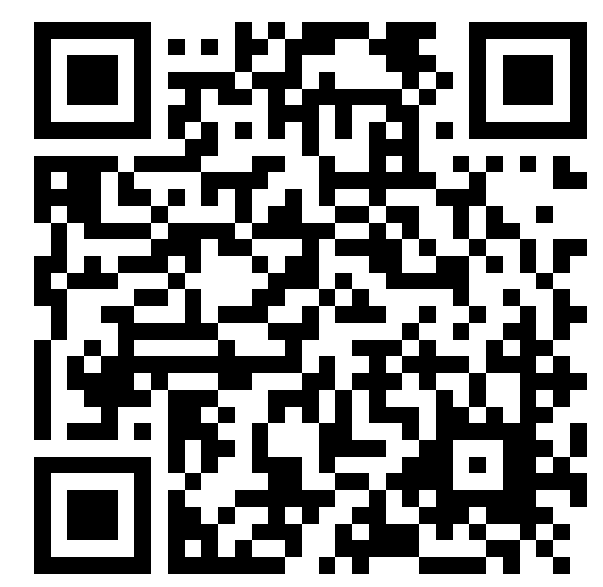

